\title{
Temperature dependent Band gap Correction model using tight-binding approach for UTB device simulations
}

This paper was downloaded from TechRxiv (https://www.techrxiv.org).

\section{LICENSE}

CC BY-NC-SA 4.0

SUBMISSION DATE / POSTED DATE

$12-01-2022$ / 14-01-2022

\section{CITATION}

Vilochan Mishra, Nalin; Solanki, Ravi; Kansal, Harshit; Medury, Aditya S (2022): Temperature dependent Band gap Correction model using tight-binding approach for UTB device simulations. TechRxiv. Preprint. https://doi.org/10.36227/techrxiv.18257963.v1

$\mathrm{DOI}$

10.36227/techrxiv.18257963.v1 


\title{
Temperature dependent Band gap Correction model using tight-binding approach for UTB device simulations
}

\author{
Nalin Vilochan Mishra, Ravi Solanki, Harshit Kansal and Aditya Sankar Medury
}

\begin{abstract}
Ultra-thin body (UTB) devices are being used in many electronic applications operating over a wide range of temperatures. The electrostatics of these devices depends on the band structure of the channel material, which varies with temperature as well as channel thickness. The semi-empirical tight binding (TB) approach is widely used for calculating channel thickness dependent band structure of any material, at a particular temperature, where TB parameters are defined. For elementary semiconductors like $\mathrm{Si}, \mathrm{Ge}$ and compound semiconductors like GaAs, these TB parameters are generally defined at only $0 \mathrm{~K}$ and $300 \mathrm{~K}$. This limits the ability of the TB approach to simulate the electrostatics of these devices at any other intermediate temperatures.

In this work, we analyze the variation of band structure for $\mathrm{Si}$, Ge and GaAs over different channel thicknesses at $0 \mathrm{~K}$ and $300 \mathrm{~K}$ (for which TB parameters are available), and show that the band curvature at the band minima has minor variation with temperature, whereas the change of band gap significantly affects the channel electrostatics. Based on this finding, we propose an approach to simulate the electrostatics of UTB devices, at any temperature between $0 \mathrm{~K}$ and $300 \mathrm{~K}$, using TB parameters defined at $0 \mathrm{~K}$, along with a suitable channel thickness and temperature dependent band gap correction.
\end{abstract}

Index Terms - Ultra-thin body (UTB), device simulation, full band simulation, tight binding, $\mathbf{s p}^{3} \mathrm{~d}^{5} \mathbf{s}^{*}$.

\section{INTRODUCTION}

In order to enable the use of ultra-thin body (UTB) devices in electronic circuits, which could find applications in space, military and consumer electronics, these devices need to be simulated over a wide range of temperatures [1]. The band structure of the channel material, which is dependent on channel thickness and operating temperature, must be taken into account [2] in order to simulate the channel electrostatics of the UTB device.

A widely used method for calculation of band structure is the tight-binding (TB) model. It is a semi-empirical scheme, which therefore requires a set of TB parameters that can replicate the band structure of the channel material at a particular temperature. In literature, TB parameters are generally defined at room temperature [3] [4] [5] or low temperature $(0 \mathrm{~K})$ [6] for elemental and compound semiconductors. Therefore, applying the TB model at any temperature other than $0 \mathrm{~K}$ and 300 $\mathrm{K}$ is not possible and this limits the applicability of the TB

N.V. Mishra, R. Solanki, H. Kansal and A. S. Medury are with the Department of Electrical Engineering and Computer Science, Indian Institute of Science Education and Research, Bhopal, MP, 462066 India e-mail: nalin20@iiserb.ac.in

Manuscript received XX, 2021; revised XX, 2021. approach for temperature dependent electrostatics simulations of UTB devices.

Recently, Zacharias et al. [7] have presented a Density Functional Theory (DFT) based approach to perform electronic band structure calculation at finite temperatures. However, DFT is a computationally cumbersome approach and is not suitable for the purpose of device simulations. Similarly, Takai et al.[8] have employed the TB molecular dynamics simulations to investigate the effect of temperature dependence on the band gap in quantum dots, which also adds an extra computational load to the TB method. On the other hand, Sawamura et al. [9] have determined the $\mathrm{sp}^{3} \mathrm{~s}^{*} \mathrm{~TB}$ parameters for various compound semiconductors at $0 \mathrm{~K}, 77 \mathrm{~K}$ and 300 $\mathrm{K}$, which again limits the applicability to only those specific temperatures. Some recent works [10] have also discussed a possible approach to get the ab initio band structure matching experiments under room temperature through the application of artificial hydrostatic strain to individual channel materials in order to mimic the effect of room temperature and also to compensate for the error from ab initio calculations. This approach involves including the effect of artificial hydrostatic strain through modification of the material's lattice constant.

In this work, we propose an approach to enable the applicability of the TB method to the simulation of electrostatics of UTB devices at any temperature between $0 \mathrm{~K}$ and 300 $\mathrm{K}$, without increasing any computational complexity. In order to do that, we firstly analyze the effects of temperature on the band structure, considering $\mathrm{Si}$, which is a widely used semiconductor, along with Ge and GaAs, having lesser and wider band gap than $\mathrm{Si}$, respectively. By showing that the band gap is the critical parameter which affects the channel electrostatics, TB parameters defined at $0 \mathrm{~K}$ along with a temperature dependent correction to the band gap will enable the TB approach to be used to simulate the device electrostatics at any other temperature between $0 \mathrm{~K}$ and $300 \mathrm{~K}$. One of the important manifestations of structural confinement of the channel material, seen in UTB devices, is the increase in band gap compared to its bulk value, which is quantified by using TB simulation parameters at $0 \mathrm{~K}$. This quantum confinement based correction along with the temperature dependent band gap correction term is used to suitably modify the temperature dependent band gap model defined for bulk semiconductors [11], thus incorporating the effect of channel thickness and temperature on the band gap. By incorporating the temperature dependent band gap variation for a particular channel thickness into the band structure calculation using the significant k-point 
selection scheme [12], a computationally efficient approach to simulate the electrostatics of UTB devices over a wide range of channel thicknesses and temperature variations, is proposed in this work.

In order to validate this approach, the integrated charge density obtained using the proposed model is compared against the integrated charge density obtained using the TB parameters given by Boykin et al. (Ge) and [3] Tan et al. (Si and GaAs) [4] at $300 \mathrm{~K}$, for various channel thicknesses and is found to be in good agreement. Also good agreement of the integrated charge density obtained from the proposed model, at a typical intermediate temperature, with TCAD simulation [13] results further validates this model.

The rest of the paper is organized as follows: In section II, firstly, the full band structure approach followed by the details of the band gap correction model is discussed. Further in this section a computationally efficient algorithm for simulating the electrostatics of UTB devices over a wide range of temperatures is proposed by incorporating a temperature dependent band gap correction model into the band structure calculation. The validation of the proposed approach with the band structure simulations at room temperature and with TCAD simulation results, at a typical intermediate temperature, is shown in section III, followed by the conclusion in section IV.

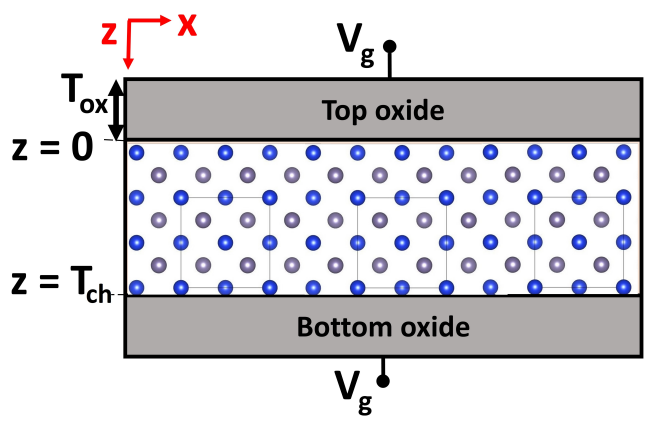

Fig. 1: Typical schematic of Ultra-thin body (UTB) double gate (DG) MOS device with Si (100) surface having 7 atomic layers (ALs).

\section{APPROACH}

In this section, we discuss an approach to use the TightBinding model for simulating the electrostatics of UTB DGMOS devices over a wide range of temperatures. In subsection II-A, a procedure is discussed to obtain the full band structure of UTB DG-MOS devices with an intrinsic (100) surface for diamond $(\mathrm{Si}, \mathrm{Ge})$ or zinc-blend (GaAs) structure, which is shown in Fig. 1. Then, the formulation based on considering TB parameters at $0 \mathrm{~K}$ along with the temperature and thickness dependent band gap correction is discussed subsequently in subsection II-B which will enable us to simulate the electrostatics of UTB devices efficiently through selecting significant $k$-points from the band structure, discussed in subsection II-C, over a wide range of channel thicknesses and temperatures.

\section{A. Full band structure approach}

The $\mathrm{sp}^{3} \mathrm{~d}^{5} \mathrm{~s}^{*}$ tight-binding (TB) model has been used to obtain the full band structure of the thin semiconductor channel [14][15]. In this model, the two centre overlap integrals considering four nearest neighbours per atom are taken into account. Infinite crystal periodicity and thus Bloch's theorem is assumed to hold good along the channel length and width directions. However, given the ultra thin nature of the channel, the crystal cannot be assumed to be periodic along the thickness direction, $z$. The TB hamiltonian has been constructed using the well known procedure outlined in [14][15][16]. The TB fitting parameters for $\mathrm{Si}$ and GaAs given by Tan et al. [4] and $\mathrm{Ge}$ given by Boykin et al. [3] are used at $300 \mathrm{~K}$, while those from Jancu et al. ( $\mathrm{Si}, \mathrm{Ge}$ and GaAs) are used at $0 \mathrm{~K}$ [6]. The top and bottom surfaces are assumed to be hydrogen passivated [17].

Given the direction of confinement being along the $z$ direction, the wave-vector $k_{z}$ in the bulk Brillioun zone (BZ) is considered to be zero, thus, reducing the $3 \mathrm{D} B Z$ to a $2 \mathrm{D}$ one. The 2D BZ is discretized along the $x$ and $y$ directions using the step size $\Delta k_{x}$ and $\Delta k_{y}$, which are taken as $0.05 \times$ $\frac{2 \pi}{a}$, where $a$ is the lattice constant of the channel material. The TB hamiltonian is assembled on each $k$-point in the irreducible $\mathrm{BZ}$ and eigenvalues of the hamiltonian plotted along $k$ space gives the band structure of the channel material.

\section{B. Band gap correction model}

In order to calculate the band structure for different channel materials, at $0 \mathrm{~K}$, it is first important to establish the correctness of using the TB model parameters proposed by Jancu et al. [6], which have been defined at low temperatures. In Table-I, we compare the band gap values obtained using Jancu's TB model parameters at a channel thickness of 50 $\mathrm{nm}$ (where quantum confinement effects are no longer seen) with the experimentally obtained bulk band gap values [18], for different channel materials, at $0 \mathrm{~K}$, showing excellent agreement.

TABLE I: Comparison of Bulk Bandgap obtained for different channel materials from experiment and Jancu's TB parameters at $\mathrm{T}=0 \mathrm{~K}$

\begin{tabular}{|c|c|c|}
\hline Material & Experiment $(\mathrm{eV})$ & Jancu $(\mathrm{eV})$ \\
\hline $\mathrm{Si}$ & 1.16929 & 1.172 \\
\hline $\mathrm{Ge}$ & 0.76918 & 0.75 \\
\hline $\mathrm{GaAs}$ & 1.521 & 1.53 \\
\hline
\end{tabular}

Through very good agreement, shown in Table-I, it may be seen that the TB model parameters proposed by Jancu et al. are indeed valid at $0 \mathrm{~K}$.

Therefore, by using the TB parameters given by Jancu et al., at $0 \mathrm{~K}$, and Boykin et al., Tan et al., at $300 \mathrm{~K}$, the band structure is obtained for $T_{c h}=2 \mathrm{~nm}$ and $10 \mathrm{~nm}$, for different channel materials and is plotted in Fig. 2. In order to match the band minima at $0 \mathrm{~K}$ and $300 \mathrm{~K}$, an offset of $\Delta E_{\min }$ is subtracted from the band structure obtained at $0 \mathrm{~K}$. This offset corresponds to a change in band gap due to temperature. From Fig. 2, it can be noted that for both $T_{c h}=2 \mathrm{~nm}$ and $10 \mathrm{~nm}$, the 
band-gap corrected band structure, at $\mathrm{T}=0 \mathrm{~K}$, in case of $\mathrm{Si}$, Ge and GaAs (anion terminated), shows very good agreement with the band structure at $\mathrm{T}=300 \mathrm{~K}$, particularly around the band minima. It may also be noted that even for GaAs (cation terminated), the band minima at $\mathrm{T}=0 \mathrm{~K}$, will align with the band minima at $\mathrm{T}=300 \mathrm{~K}$ with a band gap correction of $\Delta E_{\text {min }}=0.0841 \mathrm{eV}$ and $0.1047 \mathrm{eV}$ for $T_{c h}=2 \mathrm{~nm}$ and $T_{c h}=10$ $\mathrm{nm}$, respectively. Given the demonstration of the applicability of the temperature dependent band-gap correction approach to enable the alignment of the band minima between $\mathrm{T}=0$ $\mathrm{K}$ and $300 \mathrm{~K}$, for both GaAs (anion terminated) and GaAs (cation terminated), in this paper, we consider the case of anion termination.

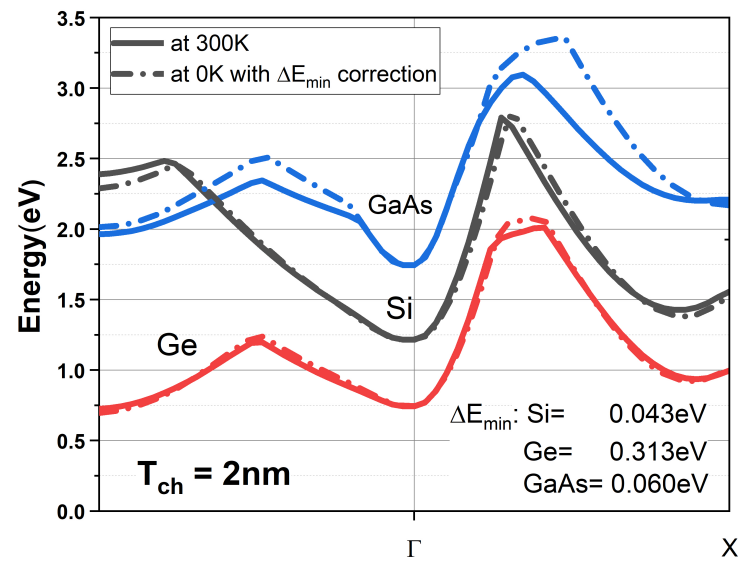

(a)

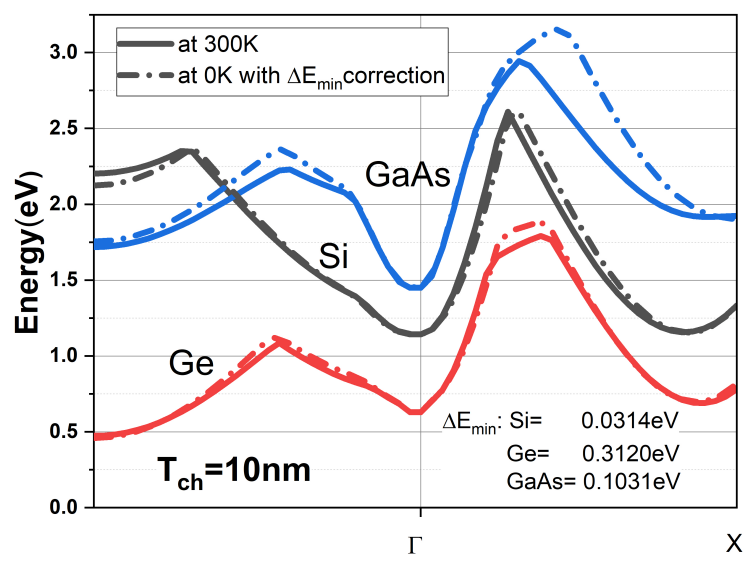

(b)

Fig. 2: The variation of band structure of $\mathrm{Si}, \mathrm{Ge}$ and $\mathrm{GaAs}$ (100) channel for (a) $T_{c h}=2 \mathrm{~nm}$ and (b) $T_{c h}=10 \mathrm{~nm}$, having $T_{o x}=1 \mathrm{~nm}$ and $V_{g}=0 \mathrm{~V}$, at $0 \mathrm{~K}$, with correction in energy to match with the band minima at $300 \mathrm{~K}$.

From Fig. 2 it is seen that the band gap of the UTB device depends on temperature $(T)$ and channel thickness $\left(T_{c h}\right)$, which can be formulated as,

$$
E_{g}\left(T_{c h}, T\right)=E_{g}(b u l k, 0)+\Delta E_{g}^{C}\left(T_{c h}, 0\right)-\Delta E_{g}^{T}\left(T_{c h}, T\right) .
$$

where, $E_{g}(b u l k, 0)$ is the bulk band gap of the semiconductor at $0 \mathrm{~K}[11]$, while $\Delta E_{g}^{C}\left(T_{c h}, 0\right)$ is the increase in band gap due to confinement in UTB devices and $\Delta E_{g}^{T}\left(T_{c h}, T\right)$ is the temperature dependent reduction in the band gap. $\Delta E_{g}^{C}\left(T_{c h}, 0\right)$ can be defined as,

$$
\Delta E_{g}^{C}\left(T_{c h}, 0\right)=E_{g}\left(T_{c h}, 0\right)-E_{g}(b u l k, 0) .
$$

where, $E_{g}\left(T_{c h}, 0\right)$ can be obtained using the TB model with parameters defined at $0 \mathrm{~K}$ [6]. Similarly, $E_{g}(b u l k, 0)$ is obtained as the band gap value at $T_{c h} \gg a_{B}$ (excitonic Bohr radius), where Quantum confinement effects can be neglected.

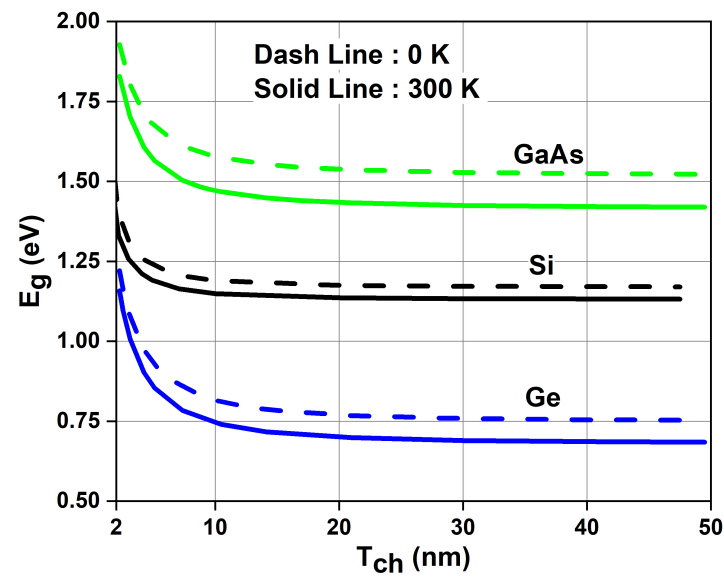

Fig. 3: The variation of band gap for $\mathrm{Si}, \mathrm{Ge}$ and GaAs channel for different $T_{c h}$, at $\mathrm{T}=0 \mathrm{~K}$ and $300 \mathrm{~K}$, when $T_{o x}=1 \mathrm{~nm}$ and $V_{g}=0 \mathrm{~V}$

$\Delta E_{g}^{T}\left(T_{c h}, T\right)$ can be defined using the well-known model of temperature dependent band gap for bulk semiconductors [11], while making one of its parameter as $T_{c h}$ dependent so as to apply it to UTB devices, as shown in Eqn.3.

$$
\Delta E_{g}^{T}\left(T_{c h}, T\right)=\frac{\alpha T^{2}}{\beta\left(T_{c h}\right)+T} .
$$

where, $\alpha$ is taken as a material constant defined by Varshni et al. [11] and $\beta$ is a parameter which is material as well as channel thickness dependent. In order to apply equation (3), the value of $\beta$, which is a function of $T_{c h}$, needs to be determined for $\mathrm{Si}, \mathrm{Ge}$ and GaAs. By rearranging Eqns. (1)-(3), for $\mathrm{T}=300 \mathrm{~K}, \beta$ is obtained, as shown in Eqn.4

$$
\beta\left(T_{c h}\right)=\frac{9 \times 10^{4} \alpha}{E_{g}\left(T_{c h}, 0\right)-E_{g}\left(T_{c h}, 300\right)}-300 .
$$

From Eqn.4, it may be seen that in order to obtain the parameter $\beta$, the values of the band gap at $\mathrm{T}=0 \mathrm{~K}$ and $300 \mathrm{~K}$ need to be determined. The required values of $E_{g}$ at various $T_{c h}$ are obtained by solving the full-band structure using parameters given by Jancu et al. [6] for $0 \mathrm{~K}$ and Boykin et al. [3], Tan et al. [4] for $300 \mathrm{~K}$. The obtained $E_{g}$ and thus obtained $\beta$ are plotted in Fig. 3 and Fig. 4, respectively, for $\mathrm{Si}, \mathrm{Ge}$ and GaAs. 


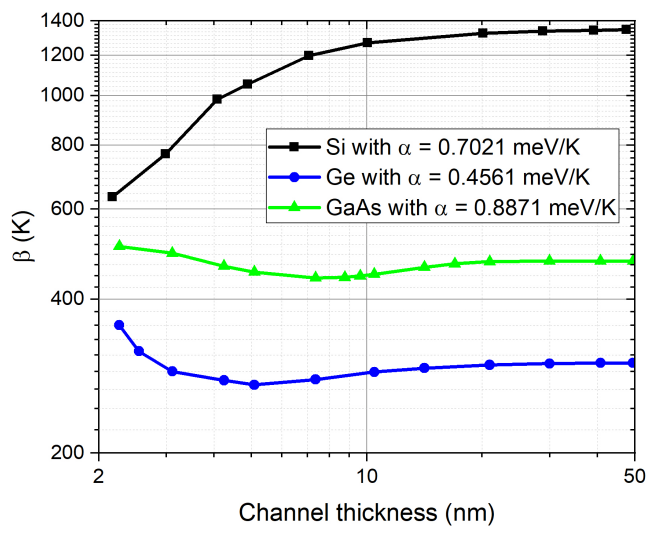

Fig. 4: The required value of the parameter $\beta$ for getting the band gap model to fit the band gap obtained from the TB model, for various channel thicknesses, at $0 \mathrm{~K}$ and $300 \mathrm{~K}$.

\section{Simulation of the Channel Electrostatics}

Given that the Full-Band structure simulation requires that the TB Hamiltonian be assembled at each k-point, this approach becomes computationally cumbersome to simulate the electrostatics of UTB MOS devices, particularly for thicker channels. Therefore, more efficient simulation of the channel electrostatics over a wide range of channel thicknesses is possible through the selection of significant k-points as proposed by Solanki et al. [12] and is utilised.

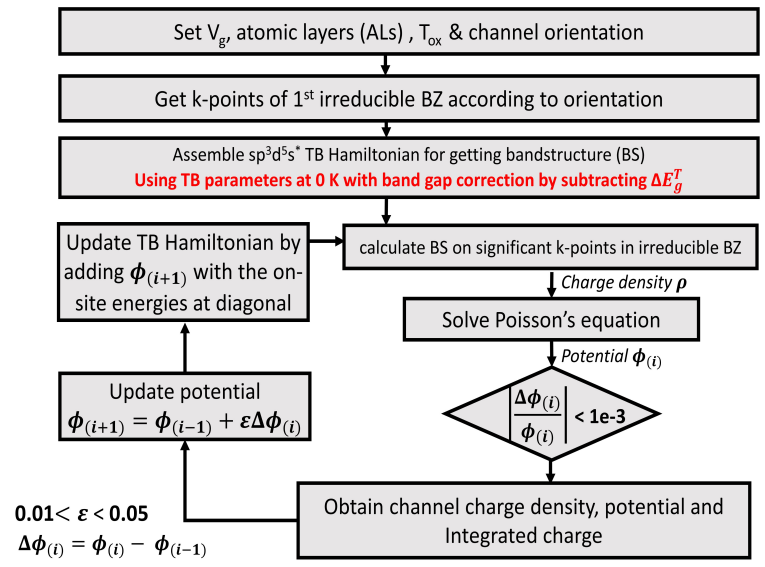

Fig. 5: Proposed algorithm for simulating the temperature dependent electrostatics of the UTB MOS device using significant $k$-points

Further, through the incorporation of a temperature dependent band gap correction term into the TB Hamiltonian, as shown in the algorithm in Fig. 5, an approach is presented to efficiently simulate the electrostatics of a UTB DG MOS device over a wide range of channel thicknesses and temperatures $(0 \mathrm{~K}$ to $300 \mathrm{~K})$.

\section{VALIDATION}

In order to demonstrate the correctness of proposed approach, the band gap model is validated over a wide range of temperatures $(0 \mathrm{~K}-300 \mathrm{~K})$. By using the correction values due to confinement and temperature (please see supplement $\mathrm{S} 1$ ), for $\mathrm{Si}, \mathrm{Ge}$ and $\mathrm{GaAs}$, over different channel thicknesses, the temperature dependent band gap curve can be extracted. The obtained temperature dependent band gap behaviour is validated through comparison with the experimental data available in literature for bulk semiconductors [18]. The proposed model is found to match well with the experimental data for $\mathrm{Si}, \mathrm{Ge}$ and GaAs at $T_{c h}=50 \mathrm{~nm}$ (equivalent to bulk) as shown in Fig. 6.

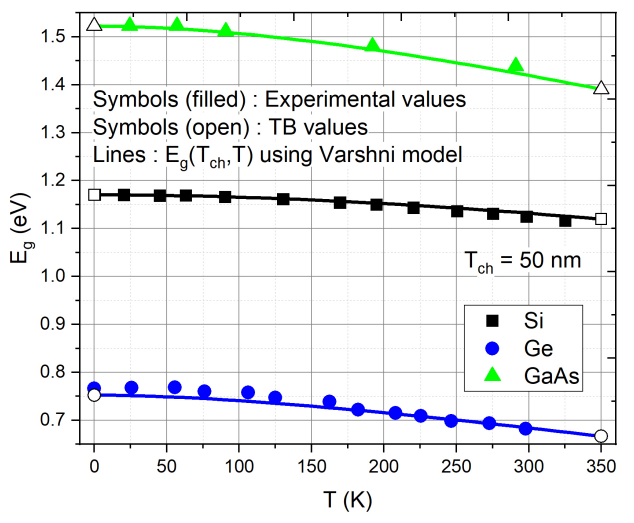

Fig. 6: Comparison of band gap versus temperature using $\Delta E_{g}^{T}\left(T_{c h}, T\right)$ model, for $T_{c h}=50 \mathrm{~nm}$, with the experimental band gap versus temperature data (filled symbols), for bulk $\mathrm{Si}, \mathrm{Ge}$ and GaAs. The model precisely follows the data points obtained using the TB model at $0 \mathrm{~K}$ and $300 \mathrm{~K}$ (open symbols).

In order to validate the proposed band gap model, at $\mathrm{T}$ $=300 \mathrm{~K}$, the integrated charge density is obtained using the algorithm shown in Fig. 5 for two typical channel thicknesses of $2 \mathrm{~nm}$ and $5 \mathrm{~nm}$, considering two cases:

1) Using Jancu's TB parameters (defined at $\mathrm{T}=0 \mathrm{~K}$ ), with a temperature dependent band gap correction term as shown in Eqn. 3.

2) Using TB parameters by Boykin et al., Tan et al. (defined at $300 \mathrm{~K}$ ) without a temperature dependent correction term.

The integrated charge densities obtained using the two cases are compared in both linear and log scale, in Fig. 7, where very good agreement may be seen. It may be noted that the efficiency of the UTB device simulation is contingent on the choice of the parameter $\eta$, which is in-turn dependent on the temperature for different channel materials (please see supplement S2).

Furthermore, we compare the integrated charge density obtained from the proposed approach with TCAD simulation results for a typical intermediate temperature of $\mathrm{T}=150 \mathrm{~K}$. We show good agreement between the proposed model and TCAD simulation results, as shown in Fig. 8. 


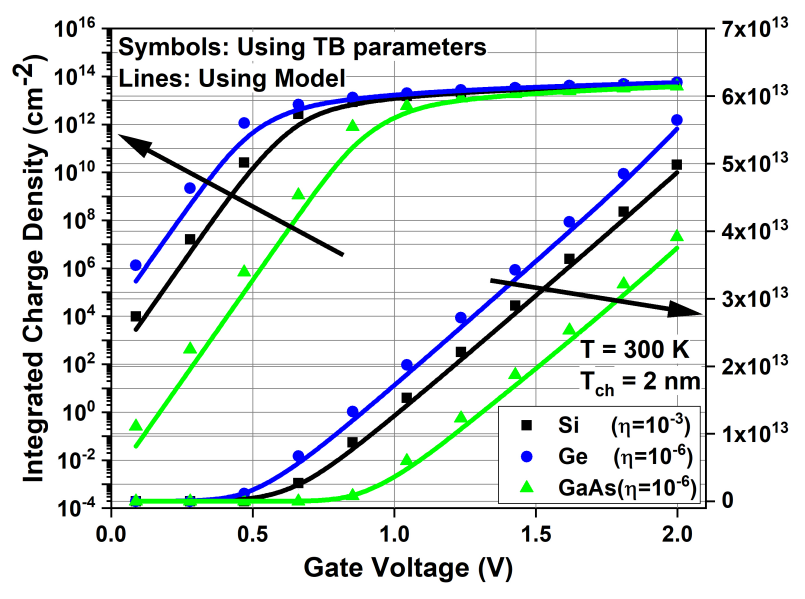

(a)

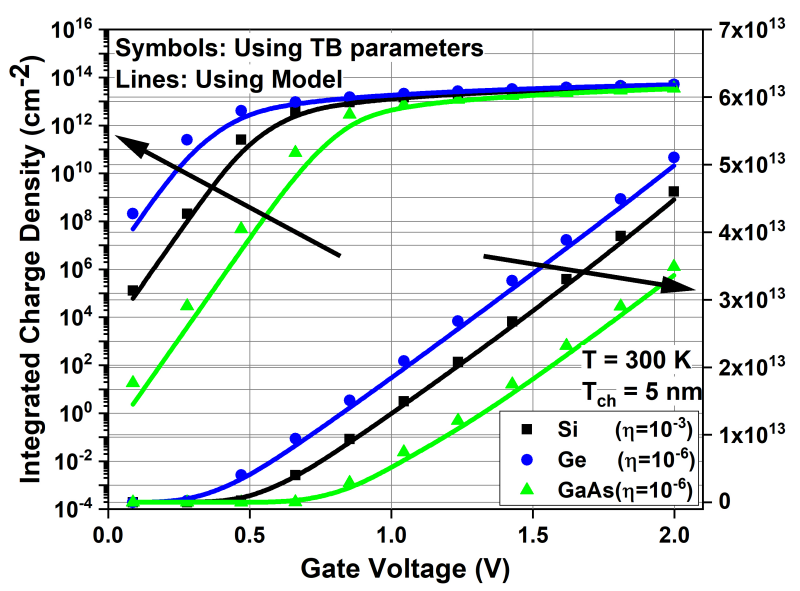

(b)

Fig. 7: Comparison of the integrated charge density obtained using the proposed approach for $\mathrm{Si}, \mathrm{Ge}$ and $\mathrm{GaAs}$ at $\mathrm{T}=300$ $\mathrm{K}$ with results obtained using TB parameters by Boykin et al. (Ge) [3] and Tan et al. ( $\mathrm{Si}, \mathrm{GaAs}$ ) [4] for (a) $T_{c h}=2 \mathrm{~nm}$ and (b) $T_{c h}=5 \mathrm{~nm}$, where $T_{o x}=1 \mathrm{~nm}$.

\section{CONCLUSION}

In this work, we present an approach for simulating the electrostatics of UTB devices using the band structure based tightbinding model valid over a wide range of device temperatures ( $0 \mathrm{~K}$ to $300 \mathrm{~K})$. We have shown that the TB parameters defined at $0 \mathrm{~K}$, can be used at any temperature between $0 \mathrm{~K}$ and 300 $\mathrm{K}$, by including the band gap correction due to temperature. The temperature and channel thickness dependence of the band gap is incorporated by modifying the well known temperature dependent band gap model used in bulk semiconductors. The proposed model is shown to be applicable over a wide range of channel materials ( $\mathrm{Si}, \mathrm{Ge}$ and $\mathrm{GaAs}$ ), channels thicknesses and device temperatures. Thus, given the availability of TB parameters at $0 \mathrm{~K}$ and $300 \mathrm{~K}$, the approach presented in this

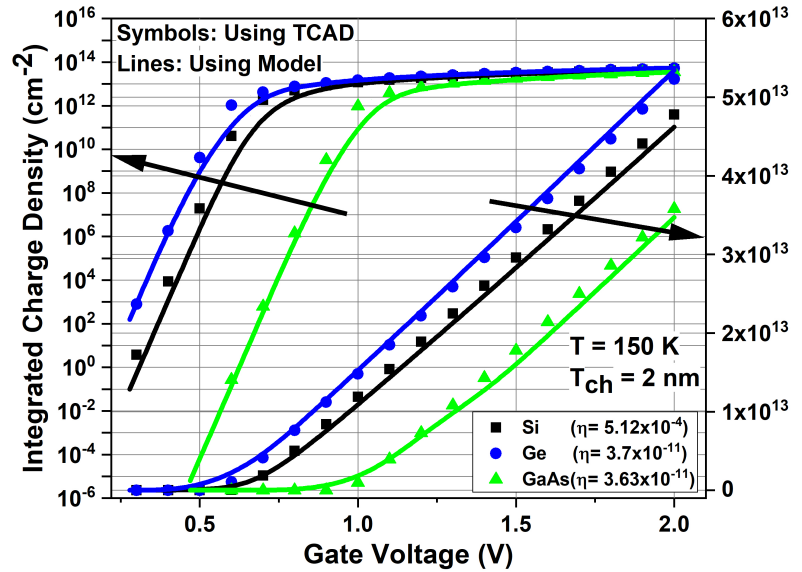

(a)

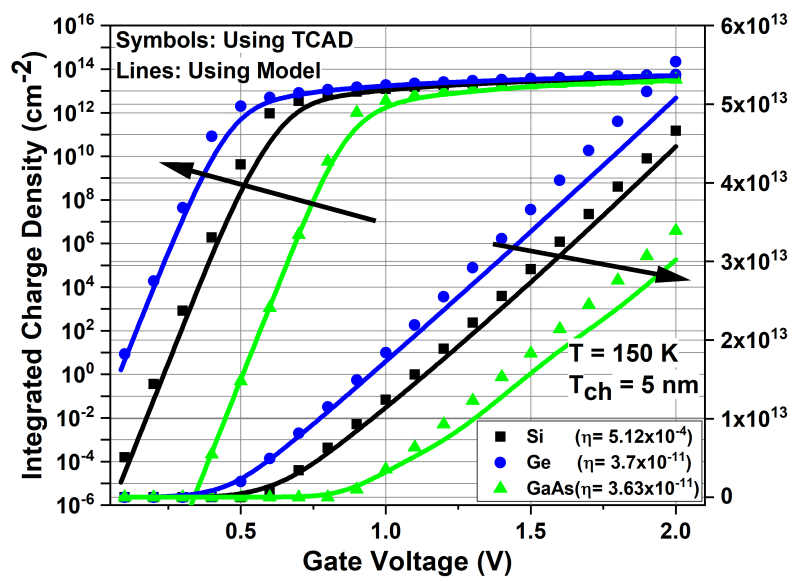

(b)

Fig. 8: Comparison of the integrated charge density obtained using the proposed approach for $\mathrm{Si}, \mathrm{Ge}$ and $\mathrm{GaAs}$ at $\mathrm{T}=150$ $\mathrm{K}$ with results obtained from TCAD simulations for (a) $T_{c h}$ $=2 \mathrm{~nm}$ and (b) $T_{c h}=5 \mathrm{~nm}$, where $T_{o x}=1 \mathrm{~nm}$.

paper can be used to effectively simulate the electrostatics of UTB devices at any intermediate temperature, without requiring $\mathrm{TB}$ parameters at that temperature.

\section{SUPPLEMENTARY MATERIAL}

S1: The bulk band gap $E_{g}(b u l k, 0)$ along with the corrections in band gap due to confinement and temperature (at $\mathrm{T}=$ $300 \mathrm{~K}$ ) for $\mathrm{Si}$, Ge and GaAs semiconductors.

S2: For efficient simulation of channel electrostatics of UTB devices, $\eta$ parameter for different channel materials is shown for various temperatures.

\section{ACKNOWLEDGMENT}

We acknowledge financial support from DST, (SERB), Government of India (Grant No: ECR/2017/000011) 


\section{REFERENCES}

[1] D.-S. Jeon and D. Burk, "A temperature-dependent soi mosfet model for high-temperature application (27 degrees c-300 degrees c)," IEEE Transactions on Electron Devices, vol. 38, no. 9, pp. 2101-2111, 1991. [Online]. Available: http://ieeexplore.ieee.org/document/83736/

[2] Y. Guo, X. Zhang, K. L. Low, K.-T. Lam, Y.-C. Yeo, and G. Liang, "Effect of body thickness on the electrical performance of ballistic nchannel gasb double-gate ultrathin-body transistor," IEEE Transactions on Electron Devices, vol. 62, no. 3, pp. 788-794, Mar 2015. [Online]. Available: http://ieeexplore.ieee.org/document/7018045/

[3] T. B. Boykin, G. Klimeck, and F. Oyafuso, "Valence band effective-mass expressions in the empirical tight-binding model applied to a si and ge parametrization," Phys. Rev. B, vol. 69, p. 115201, 2004.

[4] Y. P. Tan, M. Povolotskyi, T. Kubis, T. B. Boykin, and G. Klimeck, "Tight-binding analysis of si and gaas ultrathin bodies with subatomic wave-function resolution," Physical Review B, vol. 92, no. 8, p. 085301, 2015.

[5] T. B. Boykin, G. Klimeck, R. C. Bowen, and F. Oyafuso, "Diagonal parameter shifts due to nearest-neighbor displacements in empirical tight-binding theory," Physical Review B, vol. 66, no. 12, p. 125207 , 2002.

[6] J.-M. Jancu, R. Scholz, F. Beltram, and F. Bassani, "Empirical tightbinding calculation for cubic semiconductors: General method and material parameters," Phys. Rev. B, vol. 57, no. 11, pp. 6493-6507, 1998.

[7] M. Zacharias and F. Giustino, "Theory of the special displacement method for electronic structure calculations at finite temperature," Physical Review Research, vol. 2, no. 1, Mar 2020. [Online]. Available: https://link.aps.org/doi/10.1103/PhysRevResearch.2.013357

[8] K. Takai, M. Ikeda, T. Yamasaki, and C. Kaneta, "Size and temperature dependence of the energy gaps in si, sic and c quantum dots based on tight-binding molecular dynamics simulations," Journal of Physics Communications, vol. 1, no. 4, p. 045010, Nov 2017. [Online]. Available: https://iopscience.iop.org/article/10.1088/2399-6528/aa8db4

[9] A. Sawamura, J. Otsuka, T. Kato, and T. Kotani, "Nearest-neighbor sp $s^{*}$ tight-binding parameters based on the hybrid quasi-particle self-consistent gw method verified by modeling of type-ii superlattices," Journal of Applied Physics, vol. 121, no. 23, p. 235704, Jun 2017. [Online]. Available: http://aip.scitation.org/doi/10.1063/1.4986658

[10] Y. Tan, M. Povolotskyi, T. Kubis, T. B. Boykin, and G. Klimeck, "Transferable tight-binding model for strained group iv and iii-v materials and heterostructures," Physical Review B, vol. 94, no. 4, p. 045311, 2016.

[11] Y. P. Varshni, "Temperature dependence of the energy gap in semiconductors," physica, vol. 34, no. 1, pp. 149-154, 1967.

[12] R. Solanki, N. Mishra, and A. S. Medury, "Significant k-point selection scheme for computationally efficient band structure based utb device simulations," Semiconductor Science and Technology, 2021.

[13] S. D. U. Guide and G. Version, "Synopsys," San Jose, CA, 2008.

[14] K. Majumdar and N. Bhat, "Bandstructure effects in ultra-thin-body double-gate field effect transistor: A fullband analysis," Journal of Applied Physics, vol. 103, no. 11, p. 114503, Jun 2008. [Online]. Available: http://aip.scitation.org/doi/10.1063/1.2937186

[15] A. Rahman, G. Klimeck, and M. Lundstrom, "Novel channel materials for ballistic nanoscale mosfets-bandstructure effects," in IEEE InternationalElectron Devices Meeting, 2005. IEDM Technical Digest. IEEE, 2005, pp. 4-pp.

[16] J. C. Slater and G. F. Koster, "Simplified lcao method for the periodic potential problem," Phys. Rev., vol. 94, pp. 1498-1524, Jun 1954.

[17] S. Lee, F. Oyafuso, P. von Allmen, and G. Klimeck, "Boundary conditions for the electronic structure of finite-extent embedded semiconductor nanostructures," Phys. Rev. B, vol. 69, pp. $045316-045323$, 2004.

[18] P. Geng, W. Li, X. Zhang, X. Zhang, Y. Deng, and H. Kou, "A novel theoretical model for the temperature dependence of band gap energy in semiconductors," Journal of Physics D Applied Physics, vol. 50, no. 40, p. 40LT02, Oct 2017. [Online]. Available: https://iopscience.iop.org/article/10.1088/1361-6463/aa85ad 


\section{SUPPLEMENTARY MATERIAL}

\section{Supplement S1:}

The bulk band gap $E_{g}(b u l k, 0)$ along with the corrections in band gap due to confinement and temperature (at $\mathrm{T}=300$ $\mathrm{K})$ for $\mathrm{Si}, \mathrm{Ge}$ and $\mathrm{GaAs}$ semiconductors is shown in Fig. S1.

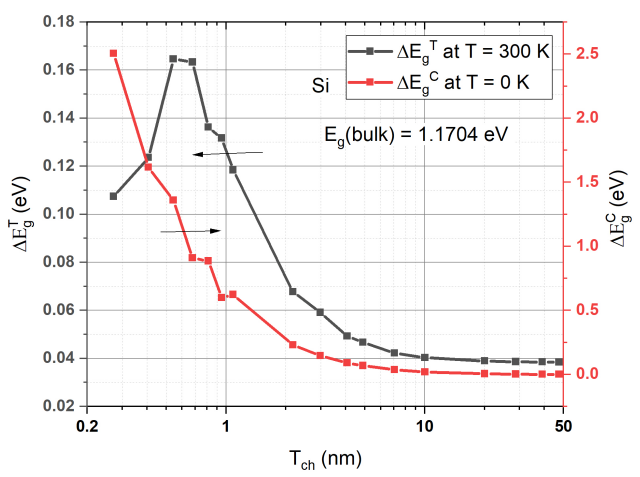

(a)

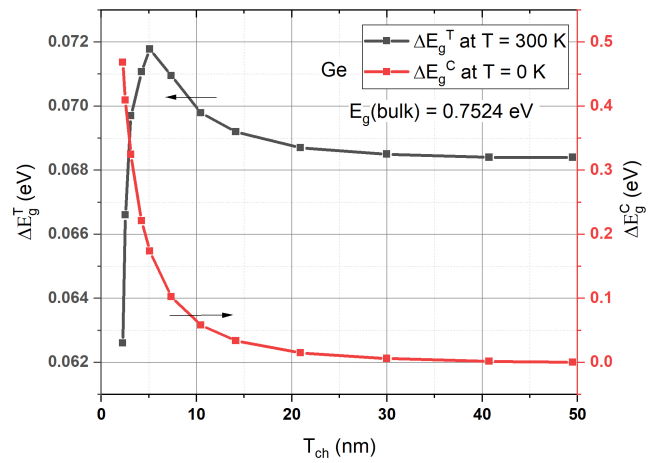

(b)

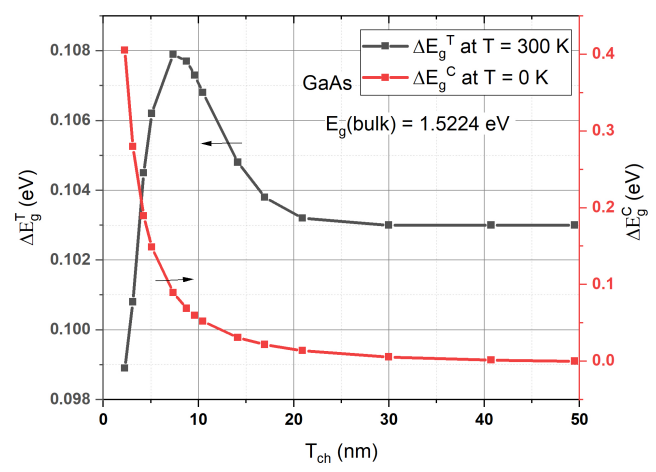

(c)

Fig. S1: The correction to the band gap due to confinement and due to temperature for (a) $\mathrm{Si}$ (b) Ge and (c) GaAs for various channel thicknesses $T_{c h}$, at $\mathrm{V}_{g}=0 \mathrm{~V}$

In the strong confinement regime, where $T_{c h}<<a_{B}$, the increase in band gap is far more significant in Si compared to
Ge and GaAs, which manifests in larger band gap variations due to temperature, also resulting in larger $\beta$ variation.

Supplement S2:

For efficient simulation of channel electrostatics for UTB devices, $k$ points are selected near band minima, where FermiDirac probabilities are significant.

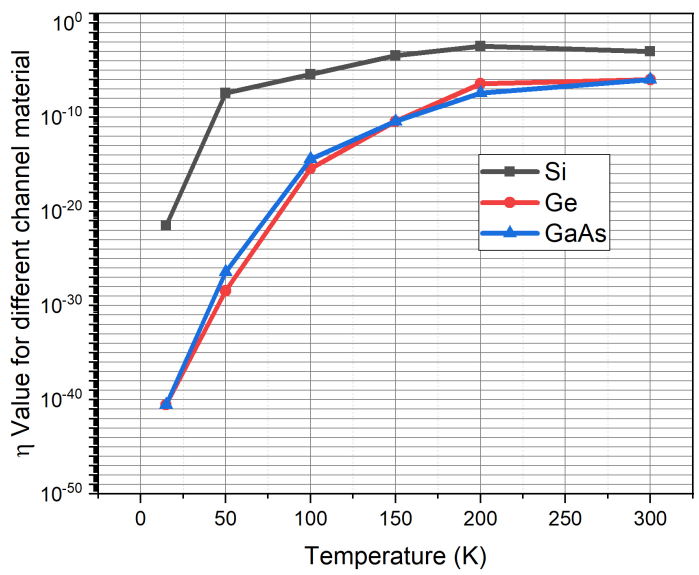

Fig. S2: The required value of the $\eta$ parameter for different channel material for getting significant $k$ points for various temperatures 